\title{
Desempenho criativo de crianças e adolescentes das cinco regiões do Brasil'
}

\author{
Tatiana de Cássia Nakano² \\ Tássia Abrão Pina \\ Priscila Zaia \\ Letícia Molina Rodrigues \\ Talita Fernanda da Silva \\ Carolina Rosa Campos
}

Pontifícia Universidade Católica de Campinas, Campinas - SP - Brasil

\begin{abstract}
Resumo: O presente estudo teve como objetivo investigar a influência do ambiente no desempenho criativo de crianças e adolescentes. Uma amostra composta por I.250 estudantes provenientes das cinco regiões do Brasil (250 de cada uma) respondeu ao teste de criatividade figural infantil. De um modo geral, os resultados apontaram para a influência da variável região de moradia nos quatro fatores avaliados pelo instrumento, assim como na maior parte das 12 características criativas nele avaliado. Em todos os fatores, perfis de desempenho bastante diferenciados foram encontrados entre as regiões brasileiras, de modo a demonstrar que, no presente estudo, as influências ambientais parecem estar influenciando a produção criativa dos estudantes avaliados. Tais resultados confirmam os apontamentos da literatura de que a criatividade não pode ser compreendida sem considerar o contexto em que o indivíduo encontra-se inserido, sendo importante considerar tal informação no momento de avaliação.
\end{abstract}

Palavras-chave: ambiente; ambiente social; criatividade; criança; adolescente.

\section{CREATIVE PERFORMANCE OF CHILDREN AND TEENS OF FIVE REGIONS OF BRAZIL}

\begin{abstract}
The present study aimed to investigate the influence of environment variable on creative performance of children and adolescents. A sample of 1250 students from five Brazilian regions ( 250 for each one) answered to the children's test of figural creativity. In general, the results suggest the influence of the variable region in all the factors measured by the instrument and in the most part of twelve characteristics. Also it is important to note that in all the factors very different performance profiles were found between regions, in order to demonstrate that in the present study, ambiental influences seems to be influencing the students' creative production. These results confirm the literature notes that creativity cannot be understood without considering the context in which the individual is inserted. This information is much important to be considered during the evaluation.
\end{abstract}

Keywords: environment; social environment; creativity; child; teenager.

1 As autoras agradecem à Fundação de Amparo à Pesquisa do Estado de São Paulo (Fapesp), ao Conselho Nacional de Desenvolvimento Científico e Tecnológico (CNPq) e ao Fundo de Apoio à Iniciação Científica (Fapic) o apoio recebido. Agradecem ainda às pesquisadoras Izabel Cavalcanti Barros Lamenha, Tatiane Lebre Dias e Áurea Lúcia M. C. M. Ferreira o auxílio na coleta de dados nas Regiões Nordeste, Centro-Oeste e Norte, respectivamente, e a Lívia Rech de Castro e Maíra Esteves Brito o auxílio na correção de parte dos instrumentos.

2 Endereço para correspondência: Tatiana de Cássia Nakano, Departamento de Pós-Graduação em Psicologia da Pontifícia Universidade Católica de Campinas, Avenida John Boyd Dunlop, s/n, Jardim Ipaussurama - Campinas - SP - Brasil. CEP: 13060-904. E-mail: tatiananakano@hotmail.com. 
RENDIMIENTO CREATIVO DE NIÑOS Y ADOLESCENTES DE CINCO REGIONES DE BRASIL

\begin{abstract}
Resumen: El presente estudio tuvo como objetivo investigar la influencia del ambiente en el desempeño creativo de niños y adolescentes. Una muestra de I. 250 estudiantes de las cinco regiones de Brasil ( 250 cada uno) respondió a la prueba figurativa de la creatividad infantil. Los resultados demuestran la influencia de la región en los cuatro factores evaluados por el instrumento, así como en la mayor parte de las características creativas. En todos los factores, perfiles de rendimiento muy diferentes fueron encontrados entre las regiones con el fin de demostrar que, en el presente estudio, las influencias ambientais parece estar influyendo en la producción creativa de los estudiantes. Estos resultados confirman las notas bibliográficas que la creatividad no se puede entender sin tener en cuenta el contexto en el que se inserta el individuo. Esa información es importante para ser considerada en el momento de la evaluación.
\end{abstract}

Palabras clave: ambiente; ambiente social; creatividad; niño; adolescente.

Segundo definição de Torrance (1965, p. 8), a criatividade pode ser definida como

[...] o processo de tornar-se sensível a problemas, deficiências, lacunas, elementos ausentes ou desarmonias; identificar as dificuldades ou os elementos faltantes nas informações; formular hipóteses, fazendo adivinhações a respeito das deficiências encontradas; testar e retestar essas hipóteses, possivelmente modificá-las e retestá-las novamente; e, finalmente, comunicar os resultados encontrados.

Considerando, desse modo, que a criatividade é um conceito complexo e multidimensional, a literatura tem demonstrado que muitos fatores podem contribuir para o desenvolvimento e a expressão da criatividade e/ou afetá-la, de modo a sugerir que a combinação de atributos cognitivos e ambientais poderiam convergir na produção de comportamentos criativos (Lubart \& Guignard, 2004). Por tal motivo, "as novas tendências no estudo da criatividade têm enfatizado a influência do contexto social, histórico e cultural no processo criativo" (Fleith, 2001, p. 55), de modo que, sob essa perspectiva, a criatividade não pode ser compreendida isolando-se o indivíduo de seu contexto.

Desde a década de 1960, estudiosos têm chamado a atenção para a importância da criatividade. Embora, nessa década e na seguinte, pesquisadores da área de criatividade até tenham reconhecido que as práticas culturais influenciam o desenvolvimento e expressão da criatividade, eles geralmente subestimaram a magnitude dessa influência. Consequentemente, concentraram sua atenção nas contribuições dos fatores cognitivos e das características pessoais, tais como motivação, personalidade, valores e capacidade de resolução de problemas para o desenvolvimento da criatividade, assim como se tornou prática comum a importação "cega" de instrumentos de medição, uma vez que tal construto tinha como pressuposto seu valor universal.

O interesse pelo meio sociocultural e histórico na criatividade começa efetivamente a ser investigado na década de 1980, ganhando impulso na década de 1990 (Rudowicz, 2003), quando os psicólogos se tornaram mais conscientes de que a cultura tem um papel muito importante no desenvolvimento da criatividade, de maneira que um número importante de investigadores começou a questionar a validade de tal 
"importação". Essa crítica levou ao desenvolvimento de investigações principalmente relacionadas com: 1. a dicotomia amplamente definida entre culturas ocidentais e orientais na percepção da criatividade; e 2. as diferenças intraculturais na percepção de criatividade (Rudowicz \& Yue, 2000).

Destaca-se, nesse sentido, que a influência do ambiente foi, durante bastante tempo, amplamente investigada sob a ótica da cultura. Assim, o contexto cultural e social começa a tornar-se mais central na visão dos pesquisadores sobre criatividade, ainda que pouco se saiba, até o momento, sobre qual seria a raiz de tais diferenças, se, por exemplo, devida a variações individuais, orientações sociais ou diferentes sistemas educacionais (Ivcevic, 2009). Especificamente em relação à cultura, Ludwig (1992) argumenta que a valorização das influências culturais é necessária para a compreensão da natureza e das manifestações da criatividade, não sendo suficiente tomá-las isoladamente. Dessa maneira, a interação entre ambos os fatores mostra-se como uma perspectiva mais válida, ainda que exista controvérsia acerca do que seria considerado "cultura". Nesse sentido, House e Javidan (2004, p. 15) definiram o conceito como "motivações, valores, crenças, identidades e interpretações de eventos significativos que resultam das experiências comuns vivenciadas pelos membros de um coletivo que são transmitidas ao longo de gerações". Assim, operariam em um determinado grupo de pessoas, dentro de uma determinada sociedade e em um determinado tempo.

Reconhece-se assim que a criatividade mostra-se socialmente definida por meio da interação entre fatores contextuais (recursos disponíveis, crenças, valores, tradições, fatores socioeconômicos, sociais, políticos, educacionais, culturais e familiares) e fatores pessoais (personalidade, inteligência, conhecimento e experiência), específicos do ambiente, os quais podem ter uma influência considerável sobre o potencial criativo do indivíduo (Fleith, 2011; Kharkhurin, 2010; Lubart, 2010; Meintjes \& Grosser, 2010). Tal visão propõe que a criatividade seria resultante de uma interação entre três forças principais: a cultura (que armazena e transmite as ideias selecionadas, os valores e as crenças para as próximas gerações), o sistema social (que seleciona quais comportamentos, valores e informações são dignos de preservação) e o indivíduo (que traz transformação para o domínio social e cultural), conforme exposto por Csikszentmihalyi (1988). Uma vez que reconhecemos que a criatividade é afetada por variáveis externas e situacionais (Watson, 2007), produto de uma interação entre esses três sistemas, ela passa a emergir como um fenômeno muito mais complexo do que aquele baseado na perspectiva individualista.

As diferenças culturais são, nesse sentido, consideradas benéficas a um povo (Saad, Damian, Benet-Martinéz, Moons, \& Robins, 2013), visto que elas tornam possível a utilização de diferentes estratégias na resolução dos mesmos problemas do dia a dia (McLean, 2009). Mesmo que uma visão de criatividade seja muitas vezes promulgada como definitiva e universal, na realidade ela se encontra localizada em um contexto particular e culturalmente específico (Westwood \& Low, 2003), com destaque para o fato de que diversos estudos já demonstraram que fatores de personalidade e aspectos cognitivos têm impacto na criatividade, mas, quando se trata de diferenças entre 
culturas, o quadro está longe de ser claro. Como consequência, sabe-se atualmente que os modelos de criatividade desenvolvidos com base na observação de uma cultura não podem ser automaticamente aplicados a outra, visto que os aspectos do sistema social podem afetar sua definição, o reconhecimento de diferentes formas de expressão, e também como e quando ideias criativas e inovações serão aceitas e adotadas (Cramond, 2008; Kaufman, Beghetto, \& Pourjalali, 2011).

Tendo como foco essa questão, pesquisas comparativas da criatividade entre diferentes países tiveram início nas décadas de 1960 e 1970, tendo sido praticamente extintas após essa época (Chen et al., 2002). Mais recentemente, estudos com o objetivo de identificar como a criatividade pode variar entre diferentes culturas, notadamente as ocidentais e orientais, voltaram a ser desenvolvidos (Sundararajan \& Averill, 2007). Como resultado, os trabalhos têm concluído que a diferença situar-se-ia, segundo Paletz e Peng (2008), na constatação de que a concepção ocidental de criatividade está principalmente preocupada com a novidade, inovação e originalidade, com base, conforme Fleith (2011), nos ideais de individualidade, democracia e liberdade. Em contraste, a concepção oriental de criatividade seria mais dinâmica, envolvendo a reutilização, quebra e reinterpretação da tradição, sendo baseada nas ideias de interdependência, coletividade, cooperação e autoritarismo. Entretanto, a resposta a essa questão acerca da existência ou não de diferenças culturais e étnicas tem se mostrado não consensual, ainda que a maior parte das pesquisas venham destacando a superioridade (Chen et al., 2002; Niu \& Sternberg, 2001; Niu, Zhang, \& Yang, 2006).

Diante da literatura disponível sobre a questão, Tsai (2012) argumenta que a maior parte dos estudos se baseia na comparação entre grupos culturais, notadamente ocidentais e orientais, destacando que outro tipo de estudo, ainda pouco explorado, volta-se à análise de subgrupos. Tal constatação ampara-se no reconhecimento de que as nações não são homogêneas, fazendo-se notar variações culturais entre grupos dentro de um mesmo país, as quais podem ser destacadas como importante ponto a ser examinado. Essa questão tem se mostrado um desafio para os pesquisadores, dada sua contribuição para o desenvolvimento de testes contextualmente adequados, a fim de que possam refletir a performance criativa em um contexto específico. Perante a tendência de implantação de modelos ocidentais nas conceituações e na maior parte das pesquisas voltadas à investigação das diferenças culturais em criatividade, dois tipos de problemas acabam sendo gerados: uma tendência a impor uma interpretação universalista sobre processos, estruturas e funções da criatividade, bem como a amplificação das diferenças presumidas (representadas sob a forma de dimensões simplistas e polarizadas), ainda que uma leitura mais cuidadosa dos dados aponte para a importância de considerar e interpretar as diferentes nuances existentes (Westwood \& Low, 2003).

Sobre essa questão, Wechsler (2001) ressalta a necessidade de identificar e promover a criatividade, respeitando as especificidades de cada nação. Pensando especificamente no caso do Brasil, poder-se-á verificar que um dos traços mais marcantes da nossa sociedade diz respeito à sua grande riqueza cultural. Em um país de dimensões 
continentais, as diferenças são muitas, sejam elas históricas, culturais ou econômicas, de modo que, por esse motivo, torna-se um desafio conhecer o grau de diversidade presente nas diversas regiões do país (Fernandes, 2001). Por tal motivo, o multiculturalismo tem sido trazido à tona nos debates mais atuais, dada a necessidade de compreender a sociedade como constituída de identidades plurais, com base na diversidade de raças, gêneros, classes sociais, padrões culturais e linguísticos, habilidades e outros marcadores (Canen \& Oliveira, 2002), destacando-se, no Brasil, as diferenças regionais presentes.

Considerando a dificuldade de mensurar a influência da cultura, dado seu grau de subjetividade, as pesquisas internacionais de comparação entre desempenho criativo de indivíduos de diferentes países usualmente têm investigado diferenças linguísticas, étnicas, de nível socioeconômico, moradia em área urbana ou rural, indivíduos monolíngues e bilíngues, diferenças de sexo, raça, origem demográfica cultural e as diferenças entre gerações (Hunsaker \& Frasier, 1999). O presente estudo, por sua vez, irá concentrar-se em outros aspectos do contexto, de maneira que a questão contextual/ ambiental a ser investigada nesta pesquisa limitar-se-á à investigação da influência da região brasileira de moradia no desempenho criativo de estudantes brasileiros. Considerando que estudos anteriores apontaram tal variável como influenciadora do desempenho criativo (Gonçalves, Fleith, \& Libório, 2011; Nakano, 2010; Nakano, Wechsler, \& Primi, 2011), a predominância de estudos transculturais envolvendo a população brasileira (Alencar, Fleith, \& Martinez, 2003; Alencar \& Martinez, 1998; Wechsler, 1985), a necessidade de desenvolvimento de pesquisas em contextos e locais específicos (Westwood \& Low, 2003), bem como o reconhecimento do impacto dos fatores contextuais no desenvolvimento das habilidades criativas, a presente pesquisa buscará responder à seguinte questão:

1. Em que extensão os fatores contextuais impactam as habilidades criativas?

Buscou-se, dessa maneira, identificar semelhanças e diferenças no desempenho criativo de participantes provenientes das cinco regiões do Brasil, com a finalidade de avaliar se as diferenças regionais podem traduzir-se em diferenças individuais na criatividade.

\section{Método}

\section{Participantes}

A amostra deste estudo foi composta por 1.250 participantes, sendo 627 do sexo feminino $(50,2 \%)$, estudantes do ensino fundamental $\left(2^{\circ}\right.$ ano $=149 ; 3^{\circ}$ ano $=134$; $4^{\circ}$ ano $=201 ; 5^{\circ}$ ano $=140 ; 6^{\circ}$ ano $=196 ; 7^{\circ}$ ano $=148 ; 8^{\circ}$ ano $=139$ e $9^{\circ}$ ano $=143$ ), oriundos de escolas públicas e particulares localizadas em cinco capitais/cidades metropolitanas brasileiras, cada uma pertencente a uma região do país. A idade mínima envolvida na amostra foi de 6 anos, e a máxima, de 17 anos ( $M=10,36$; $D P=2,35$ ), sendo importante ressaltar o cuidado em equilibrar a amostra de cada região de acordo com as variáveis sexo, série e idade. 
Quanto à representatividade da amostra por região brasileira (Norte, Sul, Sudeste, Centro-Oeste e Nordeste), deve-se mencionar que cada uma delas foi composta por 250 sujeitos, metade proveniente de cada tipo de escola (pública ou particular), escoIhidos aleatoriamente dentro da amostra de normatização do instrumento utilizado e de coletas posteriores realizadas, visando evitar a interferência da variável nível socioeconômico nos resultados.

\section{Instrumento}

O instrumento utilizado para o desenvolvimento da pesquisa foi o teste de criatividade figural infantil (Nakano et al., 2011), instrumento pelo qual os participantes são convidados a compor desenhos em três atividades, a partir de estímulos incompletos que devem ser completados sob a forma de desenhos. Na primeira atividade, é solicitado que se elabore um desenho a partir de um único estímulo pouco definido; na segunda, que se complete uma série de dez estímulos; e na terceira, que se faça o maior número de desenhos a partir do mesmo estímulo (repetido 30 vezes).

Permite a avaliação da criatividade figural por meio da pontuação de 12 características criativas (fluência, flexibilidade, elaboração, originalidade, expressão de emoção, fantasia, movimento, perspectiva incomum, perspectiva interna, uso de contexto, extensão de limites e títulos expressivos), cotadas em cada um dos desenhos / respostas fornecidos pelo participante. Os resultados são analisados em termos de quatro fatores da criatividade (Nakano \& Primi, 2012):

- Enriquecimento de ideias: refere-se à capacidade de ver a situação de uma forma mais detalhada, por um ponto de vista diferente. Agrupa as características de elaboração (nas atividades 1, 2 e 3), uso de contexto (nas atividades 2 e 3), perspectiva interna (atividades 2 e 3), perspectiva incomum (atividades 1, 2 e 3) e movimento (atividades 2 e 3 ).

- Emotividade: fator composto por características que envolvem o uso de recursos criativos ligados a uma percepção mais emocional e pelas características de expressão de emoção (atividades 2 e 3), títulos expressivos (atividades 1, 2 e 3) e fantasia (atividades 2 e 3).

- Preparação criativa: separa basicamente algumas características avaliadas na primeira atividade do teste, funcionando como um aquecimento para a realização das demais atividades. Fator composto pelas características elaboração, originalidade, uso de contexto, movimento e perspectiva interna, pertencentes à primeira atividade do teste.

- Aspectos cognitivos: composto por características criativas que fazem uso de recursos cognitivos (que envolvem a busca de soluções diferenciadas, originais e que vão além dos limites estabelecidos) e pelas características de fluência (nas atividades 2 e 3), flexibilidade (nas atividades 2 e 3), originalidade (nas atividades 2 e 3) e extensão de limites (na atividade 3 ). 


\section{Procedimentos}

A partir de um recurso presente no programa estatístico utilizado, os 250 participantes de cada região (Sudeste, Nordeste e Centro-Oeste) foram selecionados dentro da amostra de normatização do instrumento, sendo 125 de cada tipo de escola (pública ou particular). Desse modo, a partir de uma base de dados com 1.250 participantes, 750 foram selecionados aleatoriamente. Os demais participantes, provenientes das Regiões Sul e Norte, foram recrutados posteriormente à publicação do instrumento, e, a partir de amostras compostas por cerca de 500 participantes em cada uma dessas regiões, uma nova seleção aleatória, idêntica à realizada anteriormente na amostra de normatização, foi realizada. Desse modo, um total de 250 participantes de cada uma das cinco regiões foi selecionado, a fim de que os dados pudessem ser analisados no presente estudo.

A coleta de dados dessa amostra de normatização seguiu os seguintes procedimentos: após a aprovação da pesquisa pelo Comitê de Ética, foram localizados pesquisadores em diferentes regiões do país que pudessem auxiliar no contato com as escolas (escolhidas por conveniência), de modo a obter autorização para aplicação do instrumento. O Termo de Consentimento Livre e Esclarecido foi enviado aos pais ou responsáveis uma semana antes do início da coleta de dados, dada a idade dos participantes, e aplicou-se a pesquisa somente naqueles cujo documento foi devolvido assinado. Posteriormente, o teste foi aplicado de forma coletiva em sala de aula, com duração aproximada de uma hora.

Salienta-se que a pesquisadora principal esteve presente no processo de coleta de dados em todas as regiões do país, com exceção da Região Norte, onde uma pesquisadora local, devidamente treinada e instruída, realizou o processo, enviando posteriormente os instrumentos para serem corrigidos pela equipe de pesquisa (pesquisadora principal e alunos de iniciação científica). Nesse sentido, convém salientar que os avaliadores foram treinados na correção de cada uma das características cotadas, e, após o treinamento, todos corrigiram 15 instrumentos em comum, de modo a estimar a precisão dos juízes. Somente após a obtenção de índices iguais a 0,70, ou maiores, os instrumentos foram liberados para correção.

\section{Resultados}

A fim de verificar a influência da variável região de moradia no desempenho criativo, a primeira análise realizada consistiu no emprego da análise univariada da variância. Nela, a região foi considerada variável independente, e as pontuações dos sujeitos nos quatro fatores e em cada uma das características, como variáveis dependentes.

Primeiramente, a estatística descritiva, por meio da estimativa das médias e dos desvios padrão do grupo de estudantes de cada região, foi calculada para os quatro fatores e as características que os compõem, optando-se pela apresentação dos resultados de cada um dos fatores separadamente. Dessa maneira, inicialmente serão 
apresentadas as estatísticas descritivas para o fator 1 (enriquecimento de ideias), apresentadas na Tabela 1, na qual também é possível verificar os resultados da análise de variância para cada uma das características.

Tabela I. Médias, desvios padrão e significância por característica avaliada nos fatores I e 2, de acordo com a região de moradia

\begin{tabular}{|c|c|c|c|c|c|c|c|c|c|c|c|c|c|}
\hline \multicolumn{14}{|c|}{ Região } \\
\hline \multirow[b]{2}{*}{ Característica } & \multirow[b]{2}{*}{ Ativ. } & \multicolumn{2}{|c|}{$\begin{array}{l}\text { Centro-Oeste } \\
(n=250)\end{array}$} & \multicolumn{2}{|c|}{$\begin{array}{l}\text { Nordeste } \\
(n=250)\end{array}$} & \multicolumn{2}{|c|}{$\begin{array}{l}\text { Sudeste } \\
(n=250)\end{array}$} & \multicolumn{2}{|c|}{$\begin{array}{c}\text { Sul } \\
(n=250)\end{array}$} & \multicolumn{2}{|c|}{$\begin{array}{c}\text { Norte } \\
(n=250)\end{array}$} & \multicolumn{2}{|c|}{$\begin{array}{c}\text { Análise } \\
\text { univariada }\end{array}$} \\
\hline & & M & $\mathrm{DP}$ & M & DP & $M$ & DP & M & DP & $M$ & DP & $F$ & Sign. \\
\hline Fator I & & 40,34 & 19,37 & 44,67 & 20,63 & 44,89 & 22,76 & 60,86 & 35,06 & 65,88 & 58,32 & 26,31 & 0,001 \\
\hline Elaboração & I & 5,84 & 3,57 & 6,13 & 3,75 & 6,43 & 4,16 & 6,72 & 6,20 & 6,63 & 6,35 & $|, 3|$ & 0,262 \\
\hline U. contexto & I & 0,44 & 0,49 & 0,45 & 0,49 & 0,48 & 0,50 & 0,35 & 0,47 & 0,29 & 0,45 & 6,20 & 0,001 \\
\hline P. incomum & I & 0,11 & 0,32 & 0,13 & 0,33 & 0,2 & 0,33 & 0,15 & 0,36 & 0,16 & 0,38 & 1,06 & 0,374 \\
\hline Elaboração & 2 & 13,24 & 7,49 & 15,92 & 9,16 & 14,39 & 10,08 & 21,13 & 13,99 & 23,89 & 16,84 & 36,23 & 0,001 \\
\hline U. contexto & 2 & 0,86 & $\mathrm{I}, 45$ & 0,83 & 1,33 & 0,93 & $\mathrm{I}, 48$ & 0,93 & 1,69 & 0,99 & $\mathrm{I}, 70$ & 0,40 & 0,806 \\
\hline P. interna & 2 & 0,31 & 0,61 & 0,40 & 0,66 & 0,28 & 0,53 & 0,40 & 0,72 & 0,60 & 0,89 & 7,95 & 0,001 \\
\hline Movimento & 2 & 0,46 & 0,76 & 0,47 & 0,78 & 0,44 & 0,69 & 0,60 & 0,94 & 0,63 & 0,96 & 2,72 & 0,028 \\
\hline P. incomum & 2 & 0,21 & 0,51 & 0,35 & 0,64 & $0,4 \mathrm{I}$ & 0,70 & 0,34 & 0,66 & 0,55 & 0,72 & 8,51 & 0,001 \\
\hline Elaboração & 3 & 17,75 & 9,94 & 18,50 & 10,85 & 19,22 & 10,60 & 27,55 & 17,69 & 41,66 & 31,20 & 79,49 & 0,001 \\
\hline P. interna & 3 & 1,02 & 2,64 & 0,61 & 0,83 & 0,89 & 1,09 & 1,15 & 1,60 & 1,92 & 2,15 & 18,74 & 0,001 \\
\hline Movimento & 3 & 0,28 & 0,53 & 0,17 & 0,45 & 0,30 & 0,62 & 0,48 & 0,76 & 0,72 & $I, I I$ & 21,56 & 0,001 \\
\hline P. incomum & 3 & 0,61 & 0,86 & 0,70 & 1,07 & $\mathrm{I}, 00$ & $|, 3|$ & 0,81 & 1,39 & $\mathrm{I}, 85$ & 2,05 & 31,47 & 0,001 \\
\hline U. contexto & 3 & 0,08 & 0,34 & 0,11 & 0,43 & 0,18 & 0,91 & 0,26 & 0,76 & 0,92 & $\mathrm{I}, 44$ & 39,90 & 0,001 \\
\hline Fator 2 & & 2,96 & 0,30 & 3,75 & 0,30 & 2,63 & 0,30 & 3,87 & 0,31 & 4,82 & 0,39 & 3,86 & 0,001 \\
\hline Título expr. & I & 0,48 & 0,36 & 0,49 & 0,36 & 0,40 & 0,35 & 0,28 & 0,36 & 0,55 & 0,47 & 4,76 & 0,001 \\
\hline Exp. emoção & I & 0,06 & 0,10 & 0,04 & 0,10 & 0,08 & 0,10 & 0,04 & 0,16 & 0,03 & 0,13 & 1,26 & 0,281 \\
\hline Título expr. & 2 & 1,24 & 0,16 & 1,56 & 0,16 & 0,96 & 0,16 & 1,56 & 0,15 & 2,08 & 0,21 & 5,79 & 0,001 \\
\hline Exp. emoção & 2 & 0,11 & 0,06 & 0,26 & 0,06 & 0,18 & 0,06 & 0,17 & 0,04 & 0,39 & 0,08 & 4,64 & 0,001 \\
\hline Fantasia & 2 & 0,18 & 0,18 & 0,19 & 0,18 & 0,23 & 0,18 & 0,25 & 0,05 & 0,86 & 0,24 & 18,97 & 0,001 \\
\hline Título expr. & 3 & 0,70 & 0,14 & 0,92 & 0,14 & 0,63 & 0,14 & 1,05 & 0,14 & $\mathrm{I}, 42$ & 0,18 & 3,70 & 0,005 \\
\hline Exp. emoção & 3 & 0,08 & 0,03 & 0,13 & 0,03 & 0,06 & 0,03 & 0,11 & 0,03 & 0,17 & 0,04 & $\mathrm{I}, 37$ & 0,242 \\
\hline Fantasia & 3 & 0,08 & 0,02 & 0,13 & 0,02 & 0,07 & 0,02 & 0,17 & 0,02 & 0,31 & 0,03 & 8,35 & 0,001 \\
\hline
\end{tabular}

Fonte: Elaborada pelas autoras.

De acordo com os dados apresentados, pode-se verificar que a variável região de moradia exerceu influência significativa tanto na pontuação total do fator $2(F=26,31$; 
$p \leq 0,001)$ quanto na maior parte das características que o compõem (uso de contexto nas atividades 1 e 3, elaboração nas atividades 2 e 3, perspectiva interna nas atividades 2 e 3, perspectiva incomum nas atividades 2 e 3, e movimento na atividade 3). Destaca-se, nesse fator, a Região Norte, cujos estudantes apresentaram médias mais altas que os demais em todas as características.

O mesmo tipo de análise foi realizado em relação ao fator 2 (emotividade), cujos dados também encontram-se disponíveis na Tabela 1. De acordo com os dados apresentados, pode-se verificar que a variável região de moradia exerceu influência significativa tanto na pontuação total do fator $(F=3,86 ; p \leq 0,001)$ quanto em várias das características que o compõem (títulos expressivos nas atividades 1, 2 e 3, expressão de emoção na atividade 2, fantasia nas atividades 2 e 3), destacando-se novamente a Região Norte, cujos estudantes apresentaram maiores médias nas características citadas.

Mais uma vez, o mesmo tipo de análise foi empregado para analisar os resultados do fator 3 (preparação criativa), cujos resultados são apresentados na Tabela 2. Nela, podemos verificar que a variável região de moradia exerceu influência significativa na pontuação total do fator $(F=35,75$; $p \leq 0,001)$, bem como em todas as características que o compõem, com exceção de elaboração. Dessa maneira, notaram-se diferenças em relação ao desempenho na primeira atividade do teste, diferenciada das demais devido ao fato de sua proposta ser bem diferente das outras, pois solicitou-se a realização de um único desenho. Os resultados permitiram compreender que, ante a possibilidade de oferecimento de uma única resposta, estudantes de cada região apresentaram desempenhos diferenciados.

Tabela 2. Médias, desvios padrão e significância por característica avaliada nos fatores 3 e 4, de acordo com a variável região de moradia

\begin{tabular}{|c|c|c|c|c|c|c|c|c|c|c|c|c|c|}
\hline \multicolumn{14}{|c|}{ Região } \\
\hline \multirow[b]{2}{*}{ Característica } & \multirow[b]{2}{*}{ Ativ. } & \multicolumn{2}{|c|}{$\begin{array}{l}\text { Centro-Oeste } \\
(n=250)\end{array}$} & \multicolumn{2}{|c|}{$\begin{array}{l}\text { Nordeste } \\
(n=250)\end{array}$} & \multicolumn{2}{|c|}{$\begin{array}{l}\text { Sudeste } \\
(n=250)\end{array}$} & \multicolumn{2}{|c|}{$\begin{array}{c}\text { Sul } \\
(n=250)\end{array}$} & \multicolumn{2}{|c|}{$\begin{array}{c}\text { Norte } \\
(n=250)\end{array}$} & \multicolumn{2}{|c|}{$\begin{array}{c}\text { Análise } \\
\text { univariada }\end{array}$} \\
\hline & & M & DP & M & DP & $M$ & DP & $M$ & DP & $M$ & DP & $\mathrm{F}$ & Sign. \\
\hline Fator 3 & & 6,57 & 0,39 & 6,96 & 0,39 & 7,33 & 0,39 & 6,82 & 0,40 & 13,16 & 0,51 & 35,75 & 0,001 \\
\hline Elaboração & I & 5,82 & 0,30 & 6,11 & 0,31 & 6,42 & 0,30 & 6,31 & 0,31 & 7,07 & 0,40 & $|, 3|$ & 0,262 \\
\hline U. contexto & I & 0,44 & 0,03 & 0,44 & 0,03 & 0,48 & 0,03 & 0,32 & 0,03 & 0,31 & 0,04 & 6,202 & 0,001 \\
\hline Movimento & I & 0,11 & 0,11 & 0,18 & 0,11 & 0,17 & 0,11 & I,82 & 0,11 & 0,13 & 0,15 & 38,59 & 0,001 \\
\hline P. interna & I & 0,23 & 0,09 & 0,20 & 0,09 & 0,24 & 0,09 & 0,06 & 0,09 & 0,88 & 0,12 & 7,70 & 0,001 \\
\hline Títulos exp. & I & 0,48 & 0,03 & 0,49 & 0,03 & 0,40 & 0,03 & 0,33 & 0,03 & 0,55 & 0,04 & 4,76 & 0,001 \\
\hline Fator 4 & & 43,49 & 1,25 & 49,14 & 1,26 & 51,35 & $\mathrm{I}, 24$ & 47,58 & 1,27 & 66,21 & 1,63 & 32,11 & 0,001 \\
\hline Originalidade & 1 & 0,25 & 0,03 & 0,34 & 0,03 & 0,31 & 0,03 & 0,28 & 0,03 & 0,35 & 0,03 & I,44 & 0,217 \\
\hline Fluência & 2 & 9,19 & 0,16 & 9,46 & 0,17 & 9,65 & 0,16 & 7,80 & 0,17 & 9,36 & 0,22 & 18,44 & 0,001 \\
\hline
\end{tabular}


Tabela 2. Médias, desvios padrão e significância por característica avaliada nos fatores 3 e 4, de acordo com a variável região de moradia (conclusão)

\begin{tabular}{|c|c|c|c|c|c|c|c|c|c|c|c|c|c|}
\hline \multicolumn{14}{|c|}{ Região } \\
\hline \multirow[b]{2}{*}{ Característica } & \multirow[b]{2}{*}{ Ativ. } & \multicolumn{2}{|c|}{$\begin{array}{l}\text { Centro-Oeste } \\
\qquad(n=250)\end{array}$} & \multicolumn{2}{|c|}{$\begin{array}{l}\text { Nordeste } \\
(n=250)\end{array}$} & \multicolumn{2}{|c|}{$\begin{array}{l}\text { Sudeste } \\
(n=250)\end{array}$} & \multicolumn{2}{|c|}{$\begin{array}{c}\text { Sul } \\
(n=250)\end{array}$} & \multicolumn{2}{|c|}{$\begin{array}{c}\text { Norte } \\
(n=250)\end{array}$} & \multicolumn{2}{|c|}{$\begin{array}{c}\text { Análise } \\
\text { univariada }\end{array}$} \\
\hline & & M & DP & M & DP & M & DP & M & DP & $M$ & DP & $\mathrm{F}$ & Sign. \\
\hline Flexibilidade & 2 & 6,76 & 0,12 & 7,27 & 0,12 & 7,41 & 0,12 & 6,83 & 0,12 & 4,79 & 0,16 & 47,79 & 0,001 \\
\hline Originalidade & 2 & 1,36 & 0,10 & 2,72 & 0,10 & 2,88 & 0,10 & 3,35 & 0,10 & 2,67 & 0,13 & 48,41 & 0,001 \\
\hline Fluência & 3 & 11,89 & 0,49 & 13,30 & 0,50 & $|3,6|$ & 0,49 & $|I, 0|$ & 0,50 & 19,68 & 0,65 & 30,95 & 0,001 \\
\hline Flexibilidade & 3 & 9,75 & 0,30 & 10,37 & 0,31 & $|I, 0|$ & 0,30 & 8,61 & 0,31 & 9,97 & 0,40 & 8,12 & 0,001 \\
\hline Originalidade & 3 & $\mathrm{I}, 79$ & 0,24 & 2,60 & 0,24 & 3,13 & 0,23 & 5,19 & 0,24 & 10,22 & 0,31 & 137,09 & 0,001 \\
\hline Ext. limites & 3 & 2,41 & 0,24 & 3,05 & 0,24 & 3,32 & 0,24 & 3,66 & 0,24 & 10,53 & 0,31 & 117,69 & 0,001 \\
\hline
\end{tabular}

Fonte: Elaborada pelas autoras.

Tomando as características emocionais, pode-se notar ainda que a média apresentada pelos estudantes da Região Norte são significativamente mais altas que a dos demais estudantes em perspectiva interna (habilidade de prestar atenção ao interno, à dinâmica das coisas) e títulos expressivos (não só expressando a ideia básica, mas usando de habilidades como abstração).

Estudantes da Região Sudeste destacam-se em uso de contexto (capacidade de compreender o problema dentro de um universo maior, de um contexto) e os da Região Sul em movimento (atenção à dinâmica de funcionamento das coisas, e não somente à mera percepção delas), conforme definido em Nakano (2012).

Por fim, a análise dos resultados do fator 4 (aspectos cognitivos) também se encontra disponível na Tabela 2, cujos dados apontam para a influência da região de moradia nesse fator $(F=32,11 ; p \leq 0,001)$. Diferenças puderam ser notadas em relação ao uso de recursos cognitivos, que envolvem a busca de soluções que vão além dos limites estabelecidos ou ainda na tendência à busca de melhores soluções, por meio da geração de um grande número de ideias diversificadas, considerando as várias soluções possíveis para o problema que está sendo apresentado, de acordo com a descrição do fator (Nakano, 2012).

Do mesmo modo, diferenças também foram encontradas em várias das características que compõem esse fator. Estudantes da Região Norte destacam-se tanto na pontuação total no fator 4 quanto nas características de fluência, originalidade e extensão de limites, todas nas atividade 3. Já os estudantes da Região Sudeste apresentam médias mais altas nas características de fluência na atividade 2 (aptidão para produzir um grande número de ideias perante uma situação ou problema) e flexibilidade nas atividades 2 e 3 (capacidade para produzir respostas variadas e pertencentes a diferentes categorias). Na Região Sul, os estudantes destacam-se em originalidade na atividade 2 
(competência para produzir ideias que se afastam do senso comum, que permitem ir além do óbvio, quebrando o modo habitual de pensar, desenvolvendo soluções alternativas, raras e diferentes para os problemas), conforme definido por Nakano (2012).

\section{Discussão}

A análise do desempenho criativo de crianças e adolescentes provenientes das cinco regiões do Brasil em um instrumento de avaliação da criatividade figurativa apontou para a existência de diferenças regionais em todos os fatores avaliados pelo instrumento, assim como na maior parte das 12 características criativas nele avaliado. Em todos os fatores, perfis de desempenho diferenciados foram encontrados entre as regiões, de modo a demonstrar que, no presente estudo, a combinação de características de personalidade associadas à criatividade e a elementos ambientais parece estar influenciando a produção criativa dos estudantes avaliados, conforme sugerido por Lubart e Guignard (2004). Sob essa perspectiva, confirmou-se a ideia de que a criatividade não pode ser compreendida isolando-se o indivíduo de seu contexto (Fleith, 2001).

Esses dados também contribuem, de maneira mais ampla, com os resultados das pesquisas disponíveis pelo Instituto Brasileiro de Geografia e Estatística (www.ibge. gov.br) em relação às estatísticas regionais. Segundo as análises que investigam o desenvolvimento regional, a economia e a qualidade de vida das populações brasileiras, o que se pode observar é que o Brasil forma um país com padrões diferenciados (econômica, social e cultural) que refletem e são refletidos na maneira de viver dos brasileiros (principalmente em relação aos contrastes, à infraestrutura). Isso ocorre porque, de acordo com a Fundação Nacional de Desenvolvimento (www.fnde.gov.br), o Brasil possui, além de um desenvolvimento centralizado em algumas regiões (Sul e Sudeste), uma pluralidade cultural na qual há a valorização de diferentes características étnicas e influências de desigualdades socioeconômicas, fatos que pertencem à história do país, tornando-o complexo, multifacetado e com muitas contradições.

Hunsaker e Frasier (1999) destacam que o indivíduo assimila uma série de características, compartilhamento de conhecimento, comportamentos, desenvolvidos em um tempo e lugar, não se limitando a diferenças éticas, raciais ou econômicas, mas abrangendo conhecimento, comportamentos e artefatos da cultura, considerada dinâmica, visto que ela pode impactar a pessoa criativa, o lugar, produto e processo. De acordo com os autores, três focos se têm feito presentes nas investigações envolvendo a cultura: 1. o estudo da criatividade em um contexto específico (buscando responder a questões do tipo "Como a criatividade é expressa em um certo grupo cultural?"); 2. estudo da transmissão cultural via desenvolvimento criativo, investigando as formas como os jovens são ensinados a serem criativos (por meio do estudo da biografia de uma pessoa criativa em seu contexto cultural, considerando a história de seu desenvolvimento pessoal como um todo); e 3. estudos dinâmicos que têm como foco as mudanças e transformações culturais. $O$ estudo aqui apresentado baseia-se no primeiro foco, considerando a percepção de que esforços para compreender o contexto relacionado 
à criatividade podem fornecer informações importantes sobre a situação em que a criatividade aparece e sobre as pessoas que participam da situação, conforme apontado por Han (2010). Tal postura confirma a opinião defendida por Glaveanu (2010), de que a criatividade não é o produto de uma "desconexão", mas sim de "conexões" entre pessoa e meio ambiente, criador e cultura.

Tomando especificamente os resultados encontrados, foi possível perceber um desempenho superior em todos os fatores, bem como na maior parte das características criativas, obtida pelos estudantes da Região Norte. Dessa maneira, pode-se verificar uma capacidade mais desenvolvida nesses estudantes de enriquecimento das ideias, visto que, de acordo com a definição do fator 1 (Nakano et al., 2011), as habilidades avaliadas envolvem a capacidade de detalhamento, enriquecimento e contextualização com que os indivíduos tendem a visualizar uma situação, bem como a habilidade em fornecer respostas que envolvem um ponto de vista diferente. Destacam-se também, nas características emocionais (fator 2 ), aquelas que envolvem o uso de recursos criativos ligados a uma percepção mais emocional, facilitadora no processo de descoberta de uma nova ideia (Nakano et al., 2011). Também se mostraram mais criativos na primeira atividade do teste (fator 3 ) e revelaram maior facilidade em usar recursos cognitivos, que envolvem a busca de soluções diferenciadas, originais e que vão além dos limites estabelecidos (fator 4 - aspectos cognitivos).

Uma possível hipótese explicativa dessas habilidades bem desenvolvidas ampara-se na constatação de que tal região mostra-se muito rica em aspectos culturais, os quais podem ser notados, por exemplo, diante da presença de um rico folclore e várias lendas (Saci-Pererê, Vitória-Régia, Boto, Boitatá, Curupira, lara, Boi-Bumbá, entre outros), danças típicas (marujada, carimbó e cirandas), além de festas tradicionais, marcadas principalmente pela influência indígena (Festival de Parintins, Círio de Nazaré, Festa do Divino, Jerusalém da Amazônia, Folia de Reis), a qual se faz notar também no artesanato diversificado (com destaque para trançados, cestarias, cerâmica, capim dourado, produção de utensílios domésticos). Todos esses fatores contribuem para a pluralidade cultural, composta por diversas danças, crenças, comidas, festas e outros aspectos que integram a cultura dessa região. Essa pluralidade de expressões artísticas parece estar exercendo influência positiva na criatividade de seus habitantes.

Estudantes da Região Sul destacaram-se em originalidade, ou seja, competência para produzir ideias que se afastam do senso comum, que permitem ir além do óbvio, quebrando o modo habitual de pensar, desenvolvendo soluções alternativas, raras, dinâmicas e diferentes para os problemas, conforme definido em Nakano (2012). Parte dessa diversidade pode ser explicada pelas particularidades dessa região, que se diferencia bastante das demais devido ao fato de ser marcada principalmente pela influência cultural dos países europeus, acentuada pela presença de imigrantes alemães, italianos, espanhóis e portugueses, que deixaram suas marcas nos hábitos, costumes, no linguajar da população que ali habita (notada por meio das expressões típicas). Assim como a Região Norte, essa região também se marca pelas danças folclóricas 
e típicas (dança de fita, balainha, fandango), lendas (Negrinho do Pastoreio, Curupira, Boitatá, Saci) e festas tradicionais (Nossa Senhora dos Navegantes e Oktoberfest).

Estudantes da Região Sudeste destacaram-se em uso de contexto (capacidade de compreender o problema dentro de um universo maior, de um contexto), fluência (aptidão para produzir um grande número de ideias perante uma situação ou problema) e flexibilidade (capacidade para produzir respostas variadas e pertencentes a diferentes categorias). Nessa região, as manifestações culturais são muito diversificadas, fazendo-se notar grandes influências de povos indígenas, africanos, italianos e asiáticos. Dessa união de povos e culturas, surge a região com a maior população do país, a maior densidade demográfica, a economia mais desenvolvida e industrializada, concentrando mais da metade da produção do Brasil. Tais características podem ser compreendidas como estímulos às habilidades que se mostraram mais desenvolvidas nos estudantes dessa região, dada a necessidade de geração de grande número de ideias, assim como sua diversificação e contextualização, a fim de possibilitar um crescimento econômico e comercial compatível com as exigências de um mundo cada vez mais globalizado e competitivo. Também nesse sentido, Strhelau, Laban e Claro (2008) apontaram que os valores mais presentes nessa população são aqueles de reconhecimento social, ambição, busca por oportunidades de sucesso financeiro e por oportunidades de desenvolvimento de carreira.

Após essas observações, faz-se necessário ressaltar que este artigo não se propõe a investigar a influência das inúmeras variáveis geográficas no desenvolvimento da criatividade, embora a elas possa ser atribuída grande influência na expressão criativa. Buscou-se, com este estudo, enriquecer os trabalhos de investigação em amplitude nacional, uma vez que, a partir de seus resultados, objetivou-se traçar, ainda que de forma exploratória, um panorama da influência do ambiente, mais especificamente da região de moradia, no desempenho em criatividade, reforçando a constatação da presença de mistificações culturais tão abrangentes. Faz-se necessário ressaltar que estudos envolvendo amostras maiores e investigação de outros fatores, tais como método pedagógico adotado pelas escolas, motivação dos alunos na realização das atividades, faixa etária, nível socioeconômico dos participantes, escolaridade dos pais, além de outros domínios da criatividade (como a verbal) e outros instrumentos que podem ter exercido influência nos resultados obtidos, devem ser estimulados.

Dessa maneira, as diferenças encontradas poderiam ser mais bem investigadas, não podendo ser atribuídas, de forma restrita e exclusiva, à influência da região de moradia. Ressalva também deve ser feita em relação ao fato de que uma única cidade em cada região do Brasil foi selecionada como representante daquela região, ainda que um cuidado em relação à padronização do tamanho das cidades tenha sido tomado (por meio da escolha de cidades capitais ou grandes cidades metropolitanas), não se podendo desprezar a constatação de que grandes diferenças possam ser encontradas entre cidades de um mesmo Estado. Alie-se o fato de que diferentes avaliadores corrigiram os testes utilizados, de modo que, ainda que um treinamento tenha sido feito, exigindo-se um valor mínimo de concordância, essa variável pode ter exercido algum 
efeito nos resultados. Do mesmo modo, atenção deve ser dada ao fato de que foram incluídos, na amostra, estudantes de uma ampla faixa etária (crianças e adolescentes), fator que pode ter exercido influência nas diferenças ou semelhanças encontradas entre as regiões, ainda que um cuidado em equilibrar os participantes de cada região de acordo com as variáveis sexo, série e idade tenha sido tomado. Como consequência, outras variáveis, tais como maturidade, experiência escolar e habilidades cognitivas, podem ajudar a explicar os resultados obtidos, de maneira que esses aspectos merecem atenção na interpretação desses resultados.

As diferenças regionais - notadamente a lingual (sotaques e termos regionalizados), os costumes, as manifestações religiosas e a identidade da população, tomados como reflexos das diversas culturas que compõem a sua sociedade, assim como dos diferentes processos de colonização (Octaviano, 2010), diferenças criativas entre as "subculturas" (definidas por Strhelau et al. (2008), como valores, crenças, atitudes, estilos de vida, tradições, nos símbolos e nas ações de um determinado grupo, podendo esse ser determinado por um espaço demográfico) - puderam ser verificadas na amostra considerada.

Após revisão de literatura sobre a influência do ambiente e do contexto social na expressão criativa, o que se pode confirmar é que a criatividade acontece em um espaço (físico e social) e um tempo específicos (momento de desenvolvimento no nível individual e momento histórico no nível social), e o potencial individual para a criatividade seria influenciado por elementos de ambas as situações, em concordância com Ivcevic (2009). No entanto, ainda que a investigação da influência cultural na criatividade seja essencial para sua compreensão, tal influência não explica tudo. 0 que continua inexplicado é por que certos indivíduos continuam a expressar sua arte ou seu talento mesmo diante de forças sociais repressivas ou proibições (Ludwig, 1992), de modo que outras pesquisas que tenham como foco outros fatores ambientais que podem exercer influência no desempenho criativo devem ser incentivadas.

Tal constatação nos leva a uma reflexão acerca da importância do oferecimento de estímulos ambientais para a expressão da criatividade, uma vez que, ainda que os recursos pessoais para ser criativo estejam presentes, a criatividade poderá não se manifestar se as condições ambientais não se mostrarem favorecedoras. Desse modo, seu reconhecimento não dependeria somente dos esforços do próprio indivíduo, sendo de grande importância o contexto social em que ele se encontra inserido (Wechsler \& Nakano, 2002). Nesse sentido, pesquisadores de diversos países vêm apontando para a necessidade de se promoverem condições favoráveis à expressão criadora, a fim de que o potencial criativo, presente em cada indivíduo, possa se desenvolver de forma mais adequada (Kaufman et al., 2011; Torrance, 1965; Wechsler, 2008). Do mesmo modo, a criatividade poderia ser vislumbrada como um recurso por meio do qual o indivíduo pode compensar as diferenças sociais e culturais do meio em que vive.

Considerando que a literatura tem destacado que a forma mais básica de que a criatividade pode variar entre culturas está em seu próprio significado/definição, o primeiro passo na avaliação de como o contexto pode afetar a criatividade é estabelecer 
como tal construto é compreendido em diferentes culturas (Paletz \& Peng, 2008), no caso específico do presente estudo, nas regiões brasileiras. Com base em tal percepção, as diferenças regionais encontradas sugerem o início de uma zona de exploração científica. Como possibilidade de pesquisas futuras, a psicologia poderia orientar um estudo interdisciplinar sobre a relação entre ambiente, cultura e criatividade, começando pela investigação de diferenças regionais que se fazem presentes intrapaíses, partindo, em seguida, para pesquisas e experimentos cross-nacionais. Se considerarmos que a natureza da criatividade é multifacetada, a investigação da definição desse construto em indivíduos provenientes de diferentes países, culturas e regiões poderá permitir a compreensão de seus aspectos que são universais e aqueles que são específicos, considerando que as concepções e definições de criatividade em uma cultura não devem ser aplicadas sem críticas e investigação em uma outra cultura diferente, conforme defendido por Westwood e Low (2003), ainda que a natureza exata do impacto seja difícil de determinar. Faz-se notar, dessa maneira, uma forte necessidade de pesquisas desenvolvidas dentro de contextos específicos e locais, com a finalidade de determinar como a criatividade é conceituada. Do mesmo modo, faz-se importante examinar os fatores que têm influência sobre esses processos, notadamente diante da constatação de que as evidências empíricas ainda são incompletas ou deficientes, de modo a sugerir que mais trabalhos empíricos sejam necessários, pois a compreensão mais refinada das diferenças culturais na concepção e prática da criatividade pode permitir que esse potencial, presente em todos os indivíduos, seja aproveitado de forma positiva, sendo a diversidade utilizada como ferramenta para a amplificação da produção criativa brasileira.

\section{Referências}

Alencar, E. M. L. S., Fleith, D. S., \& Martínez, A. M. (2003). Obstacles to personal creativity between Brazilian and Mexican university students. The Journal of Creative Behavior, 37, 179-192.

Alencar, E. M. L. S., \& Martinez, A. M. (1998). Barreiras à expressão da criatividade entre profissionais brasileiros, cubanos e portugueses. Psicologia Escolar e Educacional, 2, 23-32.

Canen, A., \& Oliveira, A. M. A. (2002). Multiculturalismo e currículo em ação: um estudo de caso. Revista Brasileira de Educação, 21, 61-75.

Chen, C., Kasok, J., Himsel, A. J., Greenberger, E., Dong, Q., \& Xue, G. (2002). Drawings of geometric shapes: a cross-cultural examination with the consensual assessment technique. Journal of Cross-Cultural Psychology, 33, 171-187.

Cramond, B. (2008). Creativity: an international imperative for society and individual. In M. F. Morais \& S. Bahia (Orgs.). Criatividade: conceitos, necessidades e intervenção (pp.13-40). Braga: Psiquilíbrios. 
Csikszentmihalyi, M. (1988). Society, culture and person: a systems view of creativity. In R. J. Sternberg (Ed.). The nature of creativity (pp. 325-339). Cambridge, UK: Cambridge University Press.

Fernandes, C. M. (2001). Desigualdade de rendimentos e educação no Brasil: alguns indicadores de diferenças regionais. Econômica, 3(2), 231-250.

Fleith, D. S. (2001). Criatividade: novos conceitos e ideias, aplicabilidade à educação. Cadernos de Educação Especial, 17, 55-61.

Fleith, D. S. (2011). Desenvolvimento da criatividade na educação fundamental: teoria, pesquisa e prática. In S. M. Wechsler \& U. L. T. Souza (Orgs.). Criatividade e aprendizagem: caminhos e descobertas em perspectiva internacional (pp. 33-52). São Paulo: Loyola.

Glaveanu, V. P. (2010). Principles for a cultural psychology of creativity. Culture \& Psychology, 16(2), 147-163.

Gonçalves, F. C., Fleith, D. S., \& Libório, A. C. O. (2011). Criatividade em sala de aula: percepção de alunos de dois estados brasileiros. Arquivos Brasileiros de Psicologia, 63, 21-30.

Han, M. (2010). How can creativity in a social context be possible? Culture \& Psychology, 16(2), 165-173.

House, R. J., \& Javidan, M. (2004). Overview of GLOBE. In R. J. House, P. J. Hanges, M. Javian, P. Dorfman \& V. Gupta (Eds.). Leadership, culture and organizations: the GLOBE study of 62 societies (pp. 9-28). Thousand Oaks, CA: Sage.

Hunsaker, S. L., \& Frasier, M. M. (1999). Perspectives in studying creativity and cultural diversity. In A. S. Fishkin, B. Cramond \& P. Olszewski-Kubilius (Eds.). Investigating creativity in youth: research and methods (pp. 203-214). Cresskill, NJ: Hampton.

Ivcevic, Z. (2009). Creativity map: toward the next generation of theories of creativity. Psychology of Aesthetics, Creativity and the Arts, 3(1), 17-21.

Kaufman, J. C., Beghetto, R. A., \& Pourjalali, S. (2011). Criatividade na sala de aula: uma perspectiva internacional. In S. M. Wechsler \& V. L. T. Souza (Orgs.). Criatividade e aprendizagem: caminhos e descobertas em perspectiva internacional (pp. 53-72). São Paulo: Loyola.

Kharkhurin, A. V. (2010). Sociocultural differences in the relationship between bilinguism and creative potential. Journal of Cross-Cultural Psychology, 41, 776-783.

Lubart, T. (2010). Cross-cultural perspectives on creativity. In J. C. Kaufman \& R. J. Sternberg. The Cambridge handbook of creativity (pp. 265-278). Cambridge: Cambridge University Press.

Lubart, T., \& Guignard, J. H. (2004). The generality-specificity of creativity: a multivariate approach. In R. J. Sternberg, E. L. Grigorenko \& J. L. Singer (Eds.). Creativity: from potential do realization (pp. 43-56). Washington, DC: American Psychological Association. 
Ludwig, A. M. (1992). Culture and creativity. American Journal of Psychoterapy, 46(3), 454-469.

McLean, S. (2009). Stories and cosmogonies: imagining creativity beyond "nature" and "culture". Cultural Anthropology, 24(2), 213-254.

Meintjes, H., \& Grosser, M. (2010). Creative thinking in prospective teachers: the status quo and the impact of contextual factors. South African Journal of Education, 30, 361-386.

Nakano, T. C. (2010). Estilos de pensar e criar em estudantes de psicologia: diferenças regionais? Estudos e Pesquisas em Psicologia, 10(3), 682-699.

Nakano, T. C. (2012). Teste de Criatividade Figural Infantil. In C. S. Hutz (Org.). Avanços em avaliação psicológica e neuropsicológica de crianças e adolescentes II (pp. 425-451). São Paulo: Casa do Psicólogo.

Nakano, T. C., \& Primi, R. (2012). A estrutura fatorial do teste de criatividade figural infantil. Psicologia: Teoria e Pesquisa, 28(3), 275-283.

Nakano, T. C., Wechsler, S. M., \& Primi, R. (2011). Teste de criatividade figural infantil: manual técnico. São Paulo: Vetor.

Niu, W., \& Sternberg, R. (2001). Cultural influences on artistic creativity and its evaluation. International Journal of Psychology, 36, 225-241.

Niu, W., Zhang, J. X., \& Yang, Y. (2006). Does culture always matter: for creativity, yes, deductive reasoning, no! In J. C. Kaufman \& J. Baer. Creativity and reason in cognitive development (pp. 282-296). Cambridge: Cambridge University Press.

Octaviano, C. (2010). As características regionais e a identidade nacional brasileira. Revista Eletrônica de Jornalismo Científico. Recuperado em 19 fevereiro, 2013, de http://www.comciencia.br/comciencia/?section=8\&edicao=56\&id=714.

Paletz, S. B. F., \& Peng, K. (2008). Implicit theories of creativity across cultures: novelty and appropriateness in two product domains. Journal of Cross-Cultural Psychology, 39, 286-302.

Rudowicz, E. (2003). Creativity and culture: a two way interaction. Scandinavian Journal of Educational Research, 47(3), 273-290.

Rudowicz, E., \& Yue, X. D. (2000). Concepts of creativity: similarities and differences among Mailand, Hong Kong and Taiwanese Chinese. The Journal of Creative Behavior, 34(3), 175-192.

Saad, C. S., Damian, R. I., Benet-Martinéz, V., Moons, W. G., \& Robins, R. W. (2013). Multiculturalism and creativity: effects of cultural context, bicultural identity and ideational fluency. Social Psychological and Personality Science, 4, 369-375.

Strhelau, V., Laban, S., \& Claro, D. (2008). Aspectos regionais da cultura brasileira e suas implicações. [Working Paper 133/2008]. Insper, São Paulo. 
Sundararajan, L., \& Averill, J. R. (2007). Creativity in the everyday: culture, self and emotions. In R. Richards (Ed.). Everyday creativity and new view of human nature (pp. 195-220). Washington, DC: American Psychological Association.

Torrance, E. P. (1965). Rewarding creative behavior. New Jersey: Prentice Hall.

Tsai, K. C. (2012). The interplay between culture and creativity. Cross-Cultural Communication, 8(2), 15-20.

Watson, E. (2007). Who or what creates? A conceptual framework for social creativity. Human Resource Development Review, 6, 419-441.

Wechsler, S. M. (1985). A identificação do talento criativo nos Estados Unidos e no Brasil. Psicologia: Teoria e Pesquisa, 1, 140-146.

Wechsler, S. M. (2001). Criatividade na cultura brasileira: uma década de estudos. Revista Portuguesa de Psicologia: Teoria, Investigação e Prática, 6(1), 215-227.

Wechsler, S. M. (2008). Criatividade: descobrindo e encorajando (3a ed.). Campinas: Lamp, PUC-Campinas.

Wechsler, S. M., \& Nakano, T. C. (2002). Caminhos para a avaliação da criatividade: perspectiva brasileira. In R. Primi. (Org.). Temas em avaliação psicológica (pp. 103115). São Paulo: Instituto Brasileiro de Avaliação Psicológica.

Westwood, R., \& Low, D. R. (2003). The multicultural muse: culture, creativity and innovation. International Journal of Cross Cultural Management, 3, 235-259.

Submissão: 31.12 .2013

Aceitação: $1^{\circ} .9 .2014$ 